\title{
INGRESOS Y CAPITAL HUMANO: EL CASO DE BUENOS AIRES A MEDIADOS DEL SIGLO XIX
}

\author{
CARLOS NEWLAND y \\ MARIA JESUS SAN SEGUNDO \\ Universidad Carlos III
}

\section{RESUMEN}

En este trabajo se efectúa una contrastación de la teoria del capital humano, utilizando para ello una muestra correspondiente a la ciudad de Buenos Aires en 1852. Este es uno de los raros casos en que para el siglo XIX se ha obtenido la información necesaria para construir ecuaciones de ingresos. Entre las conclusiones del análisis destaca la ausencia de una relación entre salarios y alfabetización. En cambio, para los propietarios se observa una relación significativa entre educación y rentas.

Estos resultados son interpretados y comparados con 108 obtenidos en estudios referidos a otras regiones de la misma época.

\section{ABSTRACT}

In this paper a sample which gives information on males from Buenos Aires in $\mathbf{1 8 5 2}$ is used to estimate earnings equations. Some of these empirical results deserve special attention. No statistical correlation is found between salaries and literacy, but there is a significant correlation between capital owners and literacy. These results are interpreted using human capital theory. Then, they are compared with similar studies for other countries in the same period.

Numerosos estudios han analizado en los últimos años los rendimientos de las inversiones en educación, expresados en la elevación de las rentas percibidas por los individuos ${ }^{1}$. Cuando se quiere realizar ese mismo tipo de análisis para épocas anteriores se presenta la dificultad de la escasez de muestras que recojan al mismo tiempo la educación y los ingresos de la

1 En general se han obtenido rendimientos bastante elevados. Psacharopoulus (1985) obtiene tasas del 8 al $10 \%$ para los paises desarrollados y del 12 al $18 \%$ para los países en desarrollo. 
población. El conjunto de datos que se utiliza en este trabajo es uno de los raros casos en que se ha obtenido esa información. La muestra se origina en un cuestionario que tuvieron que responder en enero de 1852 más de un centenar de miembros de la milicia urbana de Buenos Aires, con el objetivo de tratar reclasificarlos para cambiar su destino a las fuerzas activas ${ }^{2}$. Esta acción se veía motivada por la necesidad de reforzar a las tropas bonaerenses que debían enfrentarse en forma inminente con el ejército aliado, comandado por Justo J. de Urquiza ${ }^{3}$. El cuestionario no sólo incluyó preguntas pertinentes a lo militar - como edad, estado de salud o habilidad para montar a caballo-, sino también alfabetismo, ocupación, ingresos (por salarios, honorarios o rentas), embriaguez, color de piel y vestimenta.

Este trabajo presenta en primer lugar una breve introducción a la realidad económica, laboral y educativa porteña, que servirá de marco a la información brindada por los encuestados. Más adelante se analiza la representatividad de la muestra respecto de la población de la ciudad. Tras una breve formulación de las ecuaciones de ingresos, se estima el rendimiento de la alfabetización y se dan algunas posibles interpretaciones de los valores obtenidos. Finalmente, se comparan los resultados con los correspondientes a otras regiones a mediados del siglo XIX.

\section{ECONOMIA Y EDUCACION PORTEÑA}

Desde la época colonial, la economia de Buenos Aires se basó fuertemente en el comercio exterior; las actividades más importantes incluían el contrabando de bienes europeos y la exportación de plata, cueros, carne salada y trigo. Ya a fines de la dominación española comenzaron a relajarse las restricciones imperiales al comercio y luego de la independencia -iniciada de facto en 1810 - se adoptaría oficialmente el librecambismo. La década del veinte fue de crecimiento económico, con una notable expansión de los saladeros, que procesaban los productos locales para su exportación. Gran Bretaña descolló enormemente en esta apertura económica: las importaciones de ese país representaban alrededor de la mitad del total registrado. En los años siguien-

2 Los casos corresponden a individuos de la milicia de la parroquia de San Telmo, junto con unos pocos de la parroquia de Catedral al Sur. Muchos de ellos hasta ese momento habian evitado el enrolamiento por sufrir alguna dolencia. Los cuestionarios se encuentran en el Archivo General de la Nación (Argentina), x 18-5-6.

3 El enfrentamiento se produjo el 3 de febrero (batalla de Caseros), siendo vencidas las fuerzas bonaerenses y derrocado el gobernador Juan Manuel de Rosas. 
tes se prosiguió con ese desarrollo, que se traducía en un incremento de las construcciones y de la actividad ganadera. En la década del cuarenta se sumó una gran expansión lanera, también destinada a la exportación a centros industriales europeos y estadounidenses. Luego del derrocamiento de Rosas en 1852 , continuó el crecimiento - simbolizado en la apertura del ferrocarril en 1857- y expresado en grandes obras públicas facilitadas por el gran aumento de los ingresos estatales, entre ellas la renovación del muelle y de la aduana. Un indicador del progreso de todo el período es el volumen alcanzado por las exportaciones de productos agropecuarios, que desde 1822 a 1857 prácticamente se triplicaron ${ }^{4}$. Esto a su vez se reflejó en la rentabilidad ganadera, que llegaba a dar un retorno anual de hasta el $30 \%$ en la década del cincuenta, rendimiento que indudablemente se transmitía al resto de la economía tanto en compensación a la tierra, como a los otros factores 5 .

En cuanto a su tamaño, Buenos Aires había sido una ciudad muy marginal en el Imperio Español. Sin embargo, desde finales del siglo XVIII mostraba un crecimiento sostenido: tenia unos 12.000 habitantes en 1750 , cifra que pasó a unos 45.000 en 1810 y a más de 92.000 en 1855 . A finales de la época colonial la ciudad había comenzado a recibir una creciente inmigración de españoles atraídos por las oportunidades laborales y los elevados salarios pagados. Esta tendencia se mantuvo, y en 1855 más del $40 \%$ de su población era foránea, destacándose los italianos (11\%), los españoles $(6 \%)$ y los franceses $(7 \%)$. No puede dudarse que el aluvión inmigratorio se debía a las altas retribuciones locales: en 1854 un ex ministro de Hacienda describía el rápido progreso económico que experimentaban los que llegaban a sus costas, quejándose al mismo tiempo de que la carestía del servicio doméstico hubiera hecho que inclusive alguien tan importante como él mismo tuviera que colaborar en las tareas de limpieza de su hogar ${ }^{6}$. Cuando viajeros comparaban a Buenos Aires con otras ciudades hispanoamericanas y europeas inmediatamente notaban lo elevado de los salarios porteños. Un chileno en 1855 los encontraba equivalentes a los pagados en California y comentaba la alta remuneración a sirvientes, carpinteros y empleados de mataderos y saladeros. Además, se sorprendía de que no hubiera visto ningún mendigo o pobre miserable durante su estadía y que todos los habitantes estuvieran bien

4 Lynch (1986), p. 625.

5 Vicuña Mackena (1936), pp. 121, 132. Sobre los altos retornos a las inversiones en actividades periodísticas, teatrales y de libreria, véase Hortelano (1936). Sabato (1989; pp. 156, 162) estima que el rendimiento anual a las inversiones en ganaderia ovina estaba en el orden del 20 al $23 \%$.

6 Roxas y Patrón a Rosas (1/4/1854), en: Raed (1980), p. 71. 
vestidos. Inclusive hallaba muy difundido el uso del apelativo "Don», que en otros lugares era reservado como un signo de preeminencia social 7.

La escolarización elemental se debía en Buenos Aires a mediados de siglo exclusivamente al sector privado, dado que Rosas habia retirado toda financiación pública al sistema escolar desde 1838. La oferta educativa constaba de un grupo relativamente grande y dinámico de escuelas particulares, en su mayor parte regentadas por mujeres. El nivel de ingresos y la demanda de enseñanza de la sociedad porteña permitió que la educación se difundiera de manera relativamente amplia: la tasa de escolarización a mediados de siglo (para niños y niñas entre 7 y 13 años) estaba en el orden del $50 \%$. A ellos se deberían agregar los que aprendían directamente en sus hogares, con la ayuda de familiares o leccionistas.

Mientras la alfabetización general llegaba en 1855 al $55 \%$, la de los nativos era superior a la de los no nativos, y la de los hombres a la de las mujeres. El $64 \%$ de los varones nacidos en Buenos Aires sabía leer, mientras que la tasa para el mismo grupo femenino era de $55^{8}$. El desarrollo del alfabetismo en Buenos Aires se hace visible a través de la demanda de publicaciones patente en la ciudad: según Domingo Faustino Sarmiento, la práctica de la lectura estaba muy difundida y se había fomentado durante la dictadura de Rosas porque sus opositores distribuian una profusión de diarios y panfletos antigubernamentales, la mayoría de ellos publicados en Montevideo ${ }^{9}$. El significativo interés por periódicos hacia 1852 es confirmada por un editor español establecido en Buenos Aires: la suscripción para su diario era lo suficientemente barata como para que hasta los más pobres lo leyeran, de ahí su elevado número de lectores pertenecientes a todas las clases sociales. El mismo año ese editor importaba de España 20.000 ejemplares de novelas de bajo precio, que se vendieron en tres meses, particularmente entre los guardias nacionales ${ }^{10}$.

\section{FIABILIDAD Y REPRESENTATIVIDAD DE LA MUESTRA}

En general puede decirse que el conjunto de entrevistas que componen la muestra utilizada refleja adecuadamente la estructura económica porteña. En

7 Vicuña Mackena (1936), p. 83. Sobre los altos salarios porteños pagados durante la década del cincuenta, véase: Sabato (1989), Pp. 92-94, y Sáenz Quesada (1982), pp. 208-213.

8 Sobre la educación en Buenos Aires, véase: Newland (1992).

9 Sarmiento (1948-1956), iliv, 44-45.

10 Hortelano (1936), pp. 215, 233. 
primer lugar se destaca en ella un buen número de individuos relacionados con la producción y comercialización de cueros, carne salada y lana, en calidad de estancieros, intermediarios, transportistas y barraqueros. Entre los oficios se encuentra a carpinteros, albañiles, zapateros, talabarteros; en la pequeña industria, a fabricantes de jabón, aceite y velas. El sector servicios, cuya importancia es natural a cualquier ciudad, incluye a comerciantes, pulperos, tenderos y escribientes.

Sobre la fiabilidad de los datos deben hacerse algunas consideraciones iniciales. En primer lugar, del conjunto de preguntas que se hicieron a los encuestados existen algunas de las que puede cuestionarse la veracidad de las respuestas. Por ejemplo, la contestación afirmativa a la pregunta de saber andar a caballo podia implicar un rápido envío al frente de batalla en la caballería. Por otro lado, casi nadie aceptaba o declaraba que era borracho, y mucho menos que la borrachera le llevaba a un comportamiento violento. Uno de los pocos que aceptaba que le gustaba tomar - Leandro Diaz-, indicaba en su declaración que "sabe tomar ginebra pero no lo hace con frecuencia, y que cuando lo ha hecho, le ha dado siempre por cantarn. Otra pregunta con posible sesgo es sobre el estado de salud, que muchos querrían presentar como muy deteriorado para evitar así la pronta movilización. Aparte de éstas, el resto de las respuestas no parece presentar graves distorsiones, como las que pedían edad, ocupación, alfabetismo, color de piel $y$ nivel de ingresos.

Para evaluar la representatividad de los encuestados se los compara en primer lugar con la población total. Debido a que la muestra incluyó únicamente a porteños, hispanoamericanos no porteños y a españoles ${ }^{11}$, se ha limitado la contrastación de las proporciones muestrales y censales (según el censo de la ciudad de 1855) a estos grupos específicos ${ }^{12}$.

$$
\text { Según censo de } 1855^{13} \text { Según muestra }
$$

Porteños

$58 \%$

$66 \%$

No porteños hispanoamericanos ..

$21 \%$

$11 \%$

Españoles

$11 \mathrm{Al}$ resto de los extranjeros - como los británicos y franceses - no se los podia enrolar por acuerdos firmados con sus naciones respectivas y por lo tanto no se ven allí reflejados. Se tuvo que dejar de lado a unos 25 encuestados (el total es de 125) al no presentar información completa.

12 Excluyendo las otras nacionalidades inmigrantes, las mujeres y a los menores de 15 años, que no aparecen reflejados en la muestra.

13 Los resultados del censo de la ciudad de 1855 fueron publicados en: Registro Estadístico 
En segundo lugar se analiza la composición de la muestra por sector económico de pertenencia, y se compara con las proporciones sobre el censo de 1855 elaboradas por Lattes y Poczter ${ }^{14}$.

\section{Según Lattes y Poczter Según muestra}

Primario

$6 \%$

$12 \%$

Secundario

$29 \%$

$21 \%$

Terciario

$64 \%$

$67 \%$

Por último se contrastan las tasas de alfabetización para los diferentes grupos:

Según Censo 185515

Según muestra

\begin{tabular}{|c|c|c|}
\hline Porteños & $64 \%$ & $77 \%$ \\
\hline No porteños hispanoamericanos .. & $52 \%$ & $54 \%$ \\
\hline Españoles $\ldots \ldots \ldots \ldots \ldots \ldots \ldots \ldots \ldots$ & $64 \%$ & $69 \%$ \\
\hline Total 16 & $62 \%$ & $73 \%$ \\
\hline
\end{tabular}

Las comparaciones realizadas denotan que la muestra no parece ser demasiado distinta al universo que representa, aunque en los porteños presenta un alfabetismo superior al general, que podria indicar un sesgo hacia los grupos de mayores ingresos. Lamentablemente no se han podido comparar la cantidad de propietarios y no propietarios, los salarios y rentas medias, al no existir datos generales para toda la ciudad. En cuanto a la edad

del Estado de Buenos Aires 5 y 6 (1855). Se tomo únicamente a los mayores de catorce años del censo, ya que la muestra se refiere a ese grupo. El cálculo de los niños menores de quince años se hizo sobre la base de las proporciones presentadas en Lattes y Poczter (1968), pp. 51-52. Para los españoles y no porteños hispanoamericanos dicho cálculo se realizó sobre la base de las proporciones dadas para la categoria general de kno nativosw.

14 Lattes y Poczter (1968), Pp. 67-68.

15 El alfabetismo presentado en el censo de 1855 se tuvo que corregir, ya que se eliminaron a los menores de siete años de las filas de los analfabetos. Ello se realizó sobre la base de las proporciones presentadas en Lattes y Poczter (1968), Pp. 51-52. Para los porteños se utilizó la correspondiente a los nativos y para los españoles y no porteños hispanoamericanos la correspondiente a los no nativos.

16 De los grupos enumerados únicamente. 
promedio, que para la muestra es de 35 años, es de 31 para la población masculina total entre 14 y 60 años ${ }^{17}$.

\section{LA TEORIA DEL CAPITAL HUMANO Y LAS ECUACIONES DE INGRESOS}

Para analizar la relación existente entre la renta de los individuos y su nivel educativo, el punto de partida habitual 18 es la estimación de una ecuación de ingresos de la forma:

$$
\ln Y_{i}=a+b E_{i}+U_{i}
$$

donde $\mathrm{Y}$ recoge los ingresos, $\mathrm{E}$ mide la educación del individuo, normalmente en años de escolarización, y $U$ es el término de error que contiene las variables no observadas.

Siguiendo a Mince ${ }^{19}$, se pueden incluir en la ecuación medidas del capital humano adquirido por los individuos en el mercado de trabajo (on-the-job training). Se especifica entonces la siguiente ecuación:

$$
\ln Y_{i}=\alpha+\beta E_{i}+\gamma_{1} E X P_{i}+\gamma_{2} E X P_{i}^{2}+\varepsilon_{i},
$$

donde $E X P_{i}$ mide la experiencia laboral del individuo, y se añade un término cuadrático para capturar la forma cóncava generalmente observada en los perfiles edad-renta. Las estimaciones obtenidas del parámetro $\beta$ se interpretan como medidas del rendimiento económico de las inversiones educativas, mientras que $\gamma_{1}$ y $\gamma_{2}$ recogen la compensación de la experiencia laboral.

Se puede argumentar que las estimaciones del retorno de la educación podrían estar sesgadas por la ausencia de medidas de habilidad de los trabajadores o de pertenencia social. Así, por ejemplo, si los individuos más inteligentes obtienen ingresos elevados y también adquieren mucha educación, en la ecuación [2], $\beta$ sobreestimará el rendimiento de las inversiones educativas. Sin embargo, las estimaciones de Griliche ${ }^{20}$ y de otros autores indican que estos sesgos no parecen ser muy importantes ${ }^{21}$.

17 Calculado a partir de Lattes y Poczter (1968), pp. 51-52.

18 Becker (1964).

19 Mincer (1974).

20 Griliches (1977).

21 Rosen (1977) pone de manifiesto las razones generales por las que $E$ es una variable endógena en las ecuaciones de ingresos y $\beta$ puede estar sesgado. La teoría del capital humano 


\section{LA MUESTRA Y LA TEORIA DEL CAPITAL HUMANO}

Con el propósito de estimar el rendimiento económico de la alfabetización en Buenos Aires en 1852, se utiliza una ecuación semilogarítmica como [2], con los valores brindados por la muestra. Las variables se definen de la siguiente manera: a) la medida de la educación de los individuos $\left(\mathrm{E}_{\mathrm{i}}\right)$ se refiere únicamente a su capacidad para leer y escribir; b) al no disponer de medidas directas de la experiencia laboral de los individuos, se han incluido su edad y edad al cuadrado en las ecuaciones.

Las retribuciones de los asalariados y cuentapropistas (de aquí en adelante denominados globalmente ACP) se basan en sus propias declaraciones, mientras que ha sido necesario estimar los ingresos anuales de los propietarios. Para ello se ha supuesto que todos trabajaban, obteniendo un rendimiento anual de su capital entre el 15 y $20 \%$, lo cual parece razonable para la época ${ }^{22}$.

En el cuadro 1 aparecen las ecuaciones de ingresos de Mincer para la muestra porteña. En las primeras dos columnas se estiman ecuaciones como [1], obteniéndose tasas de rendimiento de la alfabetización entre el 47 y el $52 \%$, según se estimen las rentas de los propietarios como el 15 o $20 \%$ de su capital. En la tercera y cuarta columna de la tabla se estiman las ecuaciones [2], que incluyen la edad de los individuos como una aproximación de su experiencia en el mercado de trabajo. Los rendimientos de la educación no varian, observándose que las rentas crecen con la edad hasta los 44-50 años. Los valores obtenidos para $\gamma_{1}$ y $\gamma_{2}$ son parecidos a los obtenidos para otros estudios, pero no están determinados con precisión. De acuerdo a estas estimaciones el rendimiento a la alfabetización en Buenos Aires aparece como muy elevado.

supone que los individuos eligen la inversión educativa que maximiza sus ingresos (el valor presente de su corriente futura de ingresos netos). En consecuencia, los individuos se autoseleccionan en las diferentes categorias educativas $y$ no se reparten aleatoriamente en ellas. La comparación de los ingresos obtenidos por personas con diferentes inversiones educativas no proporciona una estimación insesgada del rendimiento que cualquier individuo obtendría de esa inversión. Griliches (1977) y Willis y Rosen (1979) han estimado sesgos no superiores al $10 \%$ del valor estimado de $\beta$.

22 Estos supuestos sobre los rendimientos del capital sólo son relevantes cuando combinamos los ingresos de los ACP y de los propietarios (cuadro A). Al analizar los dos grupos por separado (cuadro B) no necesitamos suponer una tasa de rendimiento al capital. 


\section{CUADRO 1}

Ecuaciones de ingresos

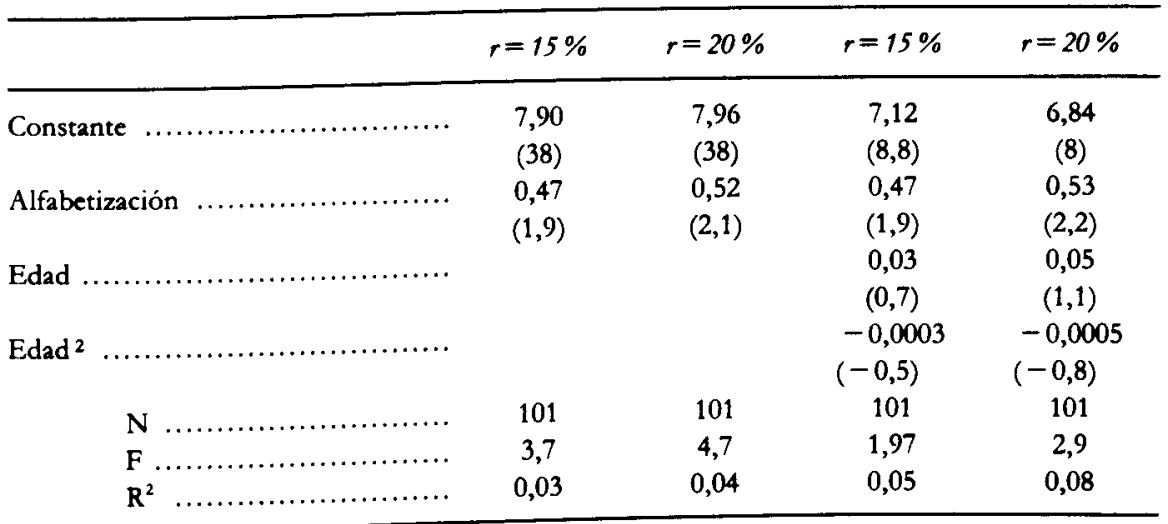

NOTA: Estimaciones por mínimos cuadrados ordinarios; estadísticos $/$ entre paréntesis.

Como la muestra está compuesta por dos grupos de individuos bien diferenciados (propietarios y no propietarios), parece conveniente investigar si las ecuaciones de ingresos son homogéneas para estos grupos. En el cuadro 2 se presentan las ecuaciones separadas para propietarios y ACP, comprobándose que no son homogéneas. La alfabetización parece ofrecer un rendimiento de cerca del $200 \%$ para los propietarios, mientras que no muestra ninguna relación estadísticamente significativa con los ingresos del otro grupo.

En las últimas columnas del cuadro 2 se estiman ecuaciones de ingresos que incluyen variables ficticias que miden la pertenencia a los sectores primario o secundario y el origen porteño o español de los individuos. La inclusión de estas variables no modifica sustancialmente los resultados. El rendimiento a la alfabetización sigue siendo muy elevado entre los propietarios $(178 \%)$ y nulo entre los ACP.

Los coeficientes de la edad y de la edad al cuadrado se estiman con poca precisión para los propietarios. Para los ACP corresponden a un perfil de rentas que alcanza su máximo hacia los 38-40 años.

En cuanto a las diferencias por sectores económicos, se observa que entre los propietarios no varían las rentas medias de los sectores secundario y terciario, mientras que hay una diferencia de ingresos del $174 \%$ a favor del sector primario. Ello sin duda es consecuencia de que en la ciudad vivian sobre todo dueños de estancias grandes y no los pequeños propietarios agropecuarios o sus encargados y peones. 


\section{CUADRO 2}

Ecuaciones de ingresos (II)

\begin{tabular}{|c|c|c|c|c|}
\hline & Propiet. & Acp. & Propiet. & $A c p$ \\
\hline Constante $\ldots \ldots \ldots \ldots$ & $\begin{array}{c}0,06 \\
(0,02)\end{array}$ & $\begin{array}{l}7,33 \\
(13)\end{array}$ & $\begin{array}{c}3,06 \\
(1)\end{array}$ & $\begin{array}{l}7,94 \\
(13)\end{array}$ \\
\hline Alfabetización $\ldots$... & $\begin{array}{l}1,92 \\
(3,2)\end{array}$ & $\begin{array}{c}-0,018 \\
(-0,1)\end{array}$ & $\begin{array}{l}1,78 \\
(2,9)\end{array}$ & $\begin{array}{c}0,01 \\
(0,06)\end{array}$ \\
\hline 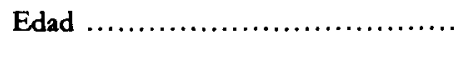 & $\begin{array}{l}0,28 \\
(1,7)\end{array}$ & $\begin{array}{c}0,063 \\
(1,8)\end{array}$ & $\begin{array}{l}0,13 \\
(0,8)\end{array}$ & $\begin{array}{l}0,06 \\
(1,7)\end{array}$ \\
\hline $\operatorname{Edad}^{2}$ & $\begin{array}{l}-0,003 \\
(-1,4)\end{array}$ & $\begin{array}{c}-0,0008 \\
(-1,7)\end{array}$ & $\begin{array}{c}-0,001 \\
(-0,6)\end{array}$ & $\begin{array}{r}-0,0008 \\
(-1,8)\end{array}$ \\
\hline Sector uno $\ldots \ldots \ldots \ldots \ldots \ldots \ldots \ldots \ldots \ldots \ldots \ldots$ & & & $\begin{array}{l}1,74 \\
(3,1)\end{array}$ & $\begin{array}{l}0,005 \\
(0,01)\end{array}$ \\
\hline Sector dos $\ldots$ & & & $\begin{array}{c}0,0006 \\
(0)\end{array}$ & $\begin{array}{l}0,52 \\
(3,1)\end{array}$ \\
\hline Porteño $\ldots \ldots \ldots \ldots \ldots \ldots \ldots \ldots \ldots \ldots \ldots \ldots \ldots \ldots$ & & & $\begin{array}{l}-0,21 \\
(-0,3)\end{array}$ & $\begin{array}{l}-0,71 \\
(-2,3)\end{array}$ \\
\hline Español $\ldots \ldots$. & & & $\begin{array}{l}0,35 \\
(0,5)\end{array}$ & $\begin{array}{r}-0,60 \\
(-1,8)\end{array}$ \\
\hline $\mathbf{N} \ldots \ldots \ldots \ldots \ldots \ldots \ldots \ldots$ & 40 & 62 & 40 & 62 \\
\hline $\mathbf{F} \ldots \ldots \ldots \ldots \ldots \ldots \ldots \ldots \ldots, \ldots$ & 6,7 & 1,2 & 5,1 & 2,7 \\
\hline $\mathbf{R}^{2} \quad \ldots \ldots \ldots \ldots \ldots \ldots \ldots \ldots \ldots \ldots$ & 0,30 & 0,05 & 0,42 & 0,25 \\
\hline
\end{tabular}

NoTA: Estimaciones por mínimos cuadrados ordinarios; estadísticos tentre paréntesis.

Entre los ACP hay un diferencial de rentas del $52 \%$ a favor del sector secundario. Dado que nuestras ecuaciones sólo incluyen una medida de alfabetización de los individuos, es posible que estas variables sectoriales recojan diferencias en habilidades o calificaciones entre los trabajadores de los diferentes sectores económicos. Si se interpretan estos coeficientes como indicativos de diferencias en rendimiento, estarian reflejando una situación transitoria que induciría la movilidad entre sectores. Por el contrario, pueden recoger un problema más permanente de segmentación del mercado de trabajo, con algún tipo de barreras a la entrada de los sectores con rentas más elevadas. El tamaño de la muestra no permite estimar ecuaciones separadas para diferentes sectores económicos. Sin embargo, la posible evidencia de distintas estructuras de ingresos tampoco apoyaria de manera concluyente la teoría de la segmentación ${ }^{23}$.

\footnotetext{
23 Heckman y Holtz (1986).
} 
Por último, el origen porteño o español de los propietarios no muestra relación con sus ingresos, mientras que en el caso de los asalariados no porteños está asociado a ingresos un 60 o $70 \%$ inferiores. El color de piel de los individuos (blanco o trigueño) no muestra ninguna relación con la renta, por lo que no se incluye en las ecuaciones estimadas.

En cuanto al ajuste estadístico de las ecuaciones, los $\mathrm{F}$ estadísticos contenidos en las tablas indican que aquellas estimadas para los asalariados no tienen una especificación aceptable. Las variables incluidas en las diferentes especificaciones no son significativas conjuntamente. Por el contrario, las ecuaciones de ingresos para los propietarios son significativas al $1 \%$.

Para analizar en detalle las relaciones entre la renta y la edad de los individuos en la muestra se presentan los cuadros 3 y 4 . En el cuadro 3 aparecen los datos para los asalariados. Los salarios crecen hasta los 40 años, pero su dispersión es tan alta, que las diferencias no son significativas. Asimismo, se observa que no hay diferencias claras entre los salarios de los alfabetos y los de los analfabetos. El salario medio se sitúa en torno a los 400 pesos al mes.

En el cuadro 4 se presentan los datos para el conjunto de los propietarios. Los alfabetos tienen un capital medio en torno a los 80.000 pesos, mientras que los escasos propietarios analfabetos presentan una media de 4.000 pesos. Los capitales medios crecen tras los 40 años, para los propietarios que saben

\section{CUADRO 3}

Salario medio ( $y$ desviación estandar)

\begin{tabular}{|c|c|c|c|}
\hline Edades & Alfabetos & Analfabetos & Todos \\
\hline \multirow[t]{2}{*}{ Menores de 20 años } & 325,3 & 296 & 318,3 \\
\hline & (187) & (118) & (174) \\
\hline \multirow[t]{2}{*}{ De 20 a 29 años .......................... } & 537,5 & 418,1 & 481,8 \\
\hline & $(336)$ & (114) & $(264)$ \\
\hline De 30 a 39 años $\ldots \ldots \ldots \ldots \ldots \ldots \ldots \ldots \ldots$ & $\begin{array}{l}516,6 \\
(209)\end{array}$ & 450 & 500 \\
\hline \multirow{2}{*}{ De 40 a 49 años $\ldots \ldots \ldots \ldots \ldots \ldots \ldots \ldots \ldots$} & $\begin{array}{l}(209) \\
379,1\end{array}$ & $(0)$ & (184) \\
\hline & $\begin{array}{l}379,1 \\
(296)\end{array}$ & 530 & 447,7 \\
\hline \multirow{2}{*}{ 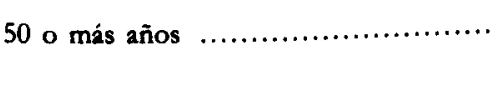 } & $\begin{array}{l}(296) \\
368,3\end{array}$ & $\begin{array}{c}(407) \\
325\end{array}$ & (359) \\
\hline & $(969)$ & $\begin{array}{c}325 \\
(117)\end{array}$ & $\begin{array}{c}351 \\
(107)\end{array}$ \\
\hline 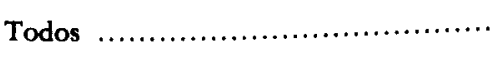 & $\begin{array}{c}398,46 \\
(251)\end{array}$ & $\begin{array}{l}400,31 \\
(243)\end{array}$ & $\begin{array}{c}399,1 \\
(245,5)\end{array}$ \\
\hline
\end{tabular}


leer y escribir. Este hecho se puede interpretar como evidencia de la productividad de la alfabetización que alcanza su máximo a esa edad. Alternativamente se podría pensar que los individuos heredaban a una edad algo tardía.

\section{CUADRO 4}

Capital medio ( $y$ desviación estándar)

\begin{tabular}{|c|c|c|c|}
\hline Edades & Alfabetos & Analfabetos & Todos \\
\hline De 20 a 29 años & $\begin{array}{c}24.625 \\
(32.197)\end{array}$ & $\begin{array}{c}4.000 \\
(0)\end{array}$ & $\begin{array}{c}22.333 \\
(31.023)\end{array}$ \\
\hline De 30 a 39 años $\ldots \ldots \ldots \ldots \ldots \ldots \ldots \ldots \ldots$ & $\begin{array}{c}48.643 \\
(47.431)\end{array}$ & $\begin{array}{c}5.333,3 \\
(943)\end{array}$ & $\begin{array}{c}35.650 \\
(44.373)\end{array}$ \\
\hline De 40 a 49 años $\ldots \ldots \ldots \ldots \ldots \ldots \ldots \ldots$ & $\begin{array}{c}160.900 \\
(172.607)\end{array}$ & - & $\begin{array}{r}160.900 \\
(172.697)\end{array}$ \\
\hline 50 o más años $\ldots \ldots \ldots \ldots \ldots$ & $\begin{array}{c}65.000 \\
(62.320)\end{array}$ & $\begin{array}{c}2.000 \\
(0)\end{array}$ & $\begin{array}{c}51.000 \\
(66.436)\end{array}$ \\
\hline Todos $\ldots \ldots \ldots \ldots$ & $\begin{array}{c}81.297 \\
(119.098)\end{array}$ & $\begin{array}{c}4.000 \\
(1.633)\end{array}$ & $\begin{array}{r}69.092,1 \\
(112.869)\end{array}$ \\
\hline
\end{tabular}

Aunque el tamaño de la muestra utilizada es reducido, el análisis reflejado en los cuadros anteriores contiene algunos resultados, el más llamativo de los cuales es que no se observa ningún rendimiento económico a la alfabetización entre los asalariados ${ }^{24}$. Este hecho admite dos interpretaciones. En primer lugar podría significar que, dado el tipo general de trabajos a desempeñar en Buenos Aires en la época, la alfabetización no influiria sobre la productividad, pudiendo realizar los analfabetos las tareas respectivas sin mayores dificultades. A esta misma conclusión llegó Tedesco ${ }^{25}$ en su estudio sobre educación y economía en Argentina a finales del siglo XIX: la estructura productiva del país no requería de una gran cantidad de personal educado para su funcionamiento al estar basada en una explotación ganadera extensiva y en un bajo desarrollo de la industria.

La segunda interpretación sería que, aun admitiendo un efecto positivo de la educación sobre la habilidad laboral en algunas actividades, la abundancia

\footnotetext{
24 Algunos peones con sueldos inferiores o equivalentes a 300 pesos mensuales, suma pequeña para la época, eran alfabetos. Tal era el caso de Juan Márquez, un porteño repartidor de pan de 40 años que ganaba uno de los salarios más bajos de la muestra: 100 pesos; igual ingreso tenía el escribiente Eduardo Larrosa.

25 Tedesco (1982).
} 
de alfabetos en 1852 hacía que su retribución diferencial fuera nula al superar ampliamente la demanda. Esta idea es compatible con la de Sabato ${ }^{26}$, quien en su análisis del mercado laboral bonaerense en la segunda mitad del siglo XIX llegó a una conclusión similar a la de Tedesco: los trabajos que requerian especialización eran muy minoritarios y la economía en general funcionaba con una escasa demanda de capacitación. Habria que concluir entonces que la demanda de alfabetización observada tendría fundamentalmente motivaciones de consumo.

Los resultados obtenidos para los propietarios son dificiles de interpretar en términos de la teoría del capital humano. Si se supone que estos individuos trabajaban, y que se ha estimado correctamente los ingresos que obtenian, se podría concluir que la alfabetización tenía un gran rendimiento para ellos. Por otro lado, es posible que el capital declarado por estos individuos proceda de herencias, y que la relación observada entre educación y riqueza se deba únicamente a la alta probabilidad de que las personas más ricas consuman educación, por razones socioculturales ${ }^{27}$.

\section{COMPARACION INTERNACIONAL Y CONCLUSIONES}

La falta de una fuerte diferenciación salarial entre trabajadores alfabetizados y no alfabetizados de Buenos Aires deja de ser sorprendente cuando se la contrasta con evidencia para otras regiones a mediados del siglo XIX. Algunos estudios han sostenido que las economías de la época, aun las más desarrolladas $e$ industrializadas, tenian una relativamente baja demanda de alfabetización, al no requerir esa habilidad una gran parte de los puestos de trabajo. Además, no es imposible pensar que, en los casos en que sí era utilizada, no tuviera posibles sustitutos ${ }^{28}$. Por otra parte, el costo de educarse podía ser considerablemente mayor que los beneficios esperados, especialmente para aquellos de estratos sociales bajos con escasas probabilidades de movilidad social ascendente. En Inglaterra, por ejemplo, a mediados del

26 Sabato (1985).

27 Los casos concretos de individuos muestran que la alfabetización no era un requisito indispensable para poseer capital: Manuel Rodríguez, propietario de carros de tráfico por un valor de 6.000 pesos, era analfabeto, lo mismo que varios dueños de carnicerias. Donde se nota una gran diferencia es en los grandes poseedores de capital, como los propietarios de estancias y los comerciantes importantes, que eran todos alfabetos. En cambio, los analfabetos que poseían capital eran transportistas (poseedores de uno o más carros), carniceros, pulperos y aguateros, es decir, contaban con capital minimo.

28 Mitch (1990). 
siglo XIX el alfabetismo no se mostraba indispensable en las tareas de una gran proporción de los trabajadores; asimismo, la Revolución Industrial, al aumentar las posibilidades de empleo, hizo que el alfabetismo presentado en algunas profesiones inclusive disminuyera al aumentar el costo de oportunidad de educarse ${ }^{29}$. Un estudio sobre la industria textil de Lancashire muestra una baja correlación entre educación y salarios; la falta de una diferencia es explicada por la baja contribución de la educación a la productividad laboral en ese sector manufacturero ${ }^{30}$.

En cuanto a Massachusetts, Nueva Inglaterra, otra región desarrollada, Field ${ }^{31}$ ha puesto en duda que la industrialización allí producida requiriera de la capacidad de leer y escribir en cantidades importantes. Sin embargo, y según una muestra, en la industria textil de ese Estado los asalariados alfabetos obtenian salarios superiores en un $17 \%$ a los analfabetos ${ }^{32}$.

La contribución económica de la educación en el siglo XIX, lejos de ser una cuestión cerrada, requiere mayores estudios y mejor información cuantitativa. Lo que quizá ya no pueda aceptarse son las afirmaciones simplistas de una correlación clara y permanente entre alfabetización y productividad, como las que hacía hacia 1850 el reformador educativo Sarmiento, quien afirmaba que la educación era el factor responsable del aumento de riqueza de un país y que haria de cada hombre un foco de producción o un taller de elaborar medios de prosperidad ${ }^{33}$. Por otra parte, deja de sorprender que cuando el inspector de escuelas Saturnino Segurola preguntó a un grupo de padres de la localidad bonaerense de Ranchos en 1830 por qué no enviaban a sus niños a la escuela, éstos respondieran que uellos no habian necesitado leer para tener platan. El informe posterior de Segurola que se referia al hecho parecía confirmar que aunque la educación era necesaria para cultivar el espíritu, no necesariamente llevaba a obtener mayores ingresos ${ }^{34}$.

29 Schofield (1973); Nicholas (1992).

30 Sanderson (1974).

31 Field (1976).

32 Aunque la diferencia es atribuida más bien a discriminación racial y de clase que a una mayor productividad (Graff, 1987, 346).

${ }^{33}$ Sarmiento (1948-1956), xii, 63.

34 AHPBA DGE Registro de Circulares 1826-34, f. 139 (Nota fechada el 12/8/1830). 


\section{BIBLIOGRAFIA}

BeCKer, G. S. (1964): Human Capital, New York.

FIELD, A. (1976): «Educational Expansion in Mid-Nineteenth-Century Massachusetts: Human-Capital Formation or Structural Reinforcement?" Harvard Educational Review, vol. 46, n. ${ }^{\circ} 4$, pp. 521-52.

GRAFF, H. (1987): The Legacies of Literacy-Continuities and Contradictions in Western Culture and Society, Boomington.

GriLICHES, Z. (1977): «Estimating the Returns to Schooling: Some Econometric Problems", Econometrica, vol. 45, n. ${ }^{\circ} 1$, pp. 1-22.

HeCKMAN, J. J., y Holtz, V. J. (1986): «An Investigation of the Labor Market Earnings of Panamian Males: Evaluating the Sources of Inequality", The Journal of Human Resources, vol. 21, n. 4 , pp. 507-542.

Hortelano, B. (1936): Memorias, Madrid.

LATTES, A., y POCZTER, R. (1968): «Muestra del censo de población de la ciudad de Buenos Aires de 1855", Documentos de Trabajo-Instituto Di Tella, n.० 54.

LYNCH, J. (1986): "The River Plate Republic from independence to the Paraguayan Wary, en Leslie Bethell ed. The Cambridge History of Latin America, Cambridge, iii, Pp. 615-676.

MINCER, J. (1974): Scbooling, Experience and Earnings, New York.

MITCH, D. (1990): «Education and economic growth: another axiom of indispensability? From human capital to human capabilities», en: G. TORTELLA, ed. Education and economic development since the Industrial Revolution, Valencia, Publicacions de la Generalitat Valenciana, pp. 29-41.

NEWLAND, C. (1992): Buenos Aires no es Pampa-La educación elemental porteña 1820-1860, Buenos Aires, Grupo Editor Latinoamericano.

Nicholas, S. J., y Nicholas, J. M. (1992): «Male Literacy, "Deskilling", and the Industrial Revolution", Journal of Interdisciplinary History, vol. xxiii, n. ${ }^{\circ} 1$, pp. 1-18.

PSACHAROPOULOS, G. (1985): «The Returns to Education: A Further International Update and Implications", The Journal of Human Resources, vol. 20, n. 4 , pp. 583604.

RAeD, J. M., ed. (1980): Cartas Inéditas de Rosas-Roxas y Patrón, Buenos Aires.

Rosen, S. (1977): "Human Capital: A Survey of Empirical Research", en R. EHRENBERG, ed., Research in Labor Economics, vol. 1, pp. 339.

SABATO, H. (1985): "La formación del mercado de trabajo en Buenos Aires, 18501890», Desarrollo Económico, vol. 24, n.*96, pp. 561-592.

- (1989): Capitalismo y Ganaderia en Buenos Aires: la fiebre del lanar 1850-1890, Buenos Aires.

SAENZ QUESADA, M. (1982): El Estado Rebelde, Buenos Aires.

SANDERSON, M. (1972): “Literacy and social mobility in the Industrial Revolution in England", Past and Present, vol. 56, pp. 75-104.

SARMiento, D. F. (1948-1956): Obras Completas, 52 vol., Buenos Aires.

SCHOFIELD, R. S. (1973): "Dimensions of Illiteracy, 1750-1850", Explorations in Economic History, vol. 10, pp. 437-454.

Tedesco, J. (1982): Educación y Sociedad en la Argentina (1880-1900), Buenos Aires. 
Vicuña Mackena, B. (1936): La Argentina en el año 1855, Buenos Aires.

WILLIS, R. J., y ROSEN, S. (1979): «Education and Self-Selection», Journal of Political Economy, vol. 87, n. 5 (octubre), pp. S7-S36. 